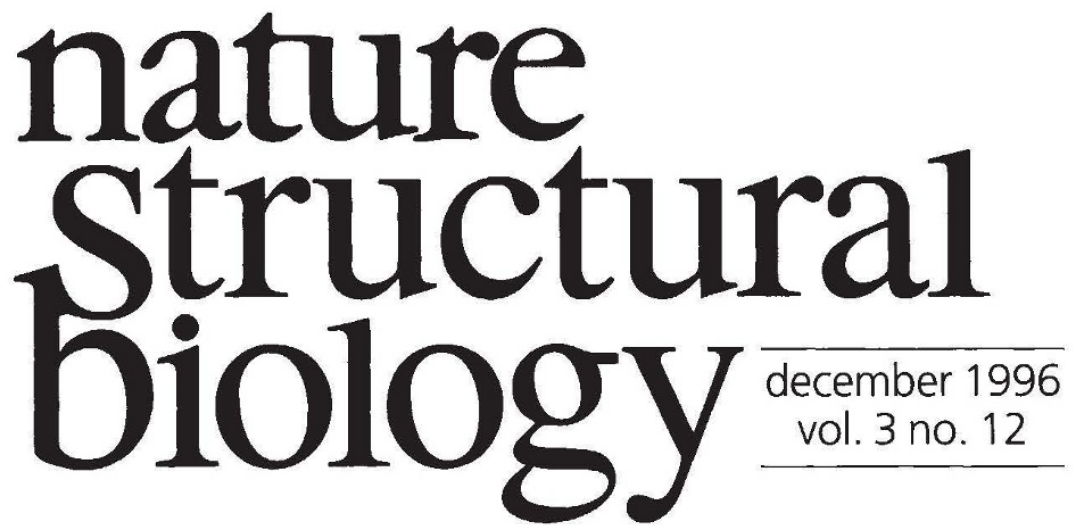

\title{
Dodging the rocks
}

Analysis of some of the oldest known sedimentary rocks on the planet has pushed back the date of the first appearance of life on Earth by some 300-400 million years- to $3.85 \mathrm{Gyr}$ before present $(\mathrm{bp})^{1}$. These results suggest that the evolution of life may have overlapped with a period of the Solar System's history known as the 'late heavy bombardment' - a time when, it is thought, the Earth was subjected to numerous massive impacts by products of planetary accretion, any number of which may have had the potential to sterilize the entire planet.

The earliest known microfossils hail from Western Australia and are thought to be some $\sim 3.5 \mathrm{Gyr}$ old, exhibiting such complex features as cell wall formation, evidence of motility, phototaxis and mat formation-suggesting that these organisms already had a substantial evolutionary history. The search for even earlier evidence of life is complicated by the fact that older crustal rocks have been subjected to intense metamorphism, which would destroy any fragile microfossils. To get around this problem, Mojzsis et al. ${ }^{1}$ analysed carbonaceous inclusions in grains of apatite found within the 3.85 Gyr old sedimentary rocks in Western Greenland, to pursue geochemical evidence of biological activity. Using a sensitive measuring technique for the ${ }^{12} \mathrm{C}$ and ${ }^{13} \mathrm{C}$ isotopes of carbon, the authors show that these inclusions are enriched for ${ }^{12} \mathrm{C}$, a strong indication of the presence of life.

How did this primordial ecosystem survive the apparently hair-raising trials of the late heavy bombardment? The flux of material hammering the Earth during the Hadean (pre 3.8 Gyr) and early Archean (3.8-3.4 Gyr) periods can be estimated from analysis of the impact record of the Moon ${ }^{2,3}$. For example, the Man-in-the-Moon's right eye-the Imbrium basin-was the result of an impact of a body some $\sim 130 \mathrm{~km}$ in diameter and with a mass of $7.5 \times 10^{18} \mathrm{~kg}$, around 3.85-3.95 Gyr bp. The Earth is a much bigger celestial target than the Moon, and so would tend to be hit by about 20 times as many projectiles, some of which could be expected to be much larger than the Imbrium object.

The consequences of such an impact on any newly minted planetary ecosystem would have been dramatic. A lump of rock $440 \mathrm{~km}$ diameter and travelling at $17 \mathrm{~km} \mathrm{~s}^{-1}$ would, on collision with the Earth, impart enough energy to the planet's ocean to completely vaporize it; rock vapor blasted into the heavens would have an effective temperature of $2,000 \mathrm{~K}$ and would initially ablate the surface of the ocean-the vapour would then rain out of the sky to form a layer of rock raindrops some $300 \mathrm{~m}$ deep on the ocean floor, at which point half the ocean would be high-pressure steam in the atmosphere at $\sim 1,500 \mathrm{~K}$; 
finally the remnants of the ocean would completely evaporate. Cooling would take at least 2000 years. Impact of a much smaller, $190 \mathrm{~km}$ diameter object would evaporate the $200 \mathrm{~m}$ deep photic zone of the ocean, with adverse conditions persisting in the atmosphere and ocean for a mere 300 years-such an impact is thought to have been quite probable as late as $3.8 \mathrm{Gyr}$ bp.

Sterilization of the Earth seems quite likely in the event of an impact massive enough to completely evaporate the ocean: any primeval ecosystems would have certainly been reset to zero. Even under relatively

\section{The Moon is a harsh mistress}

The collision of a Mars-sized planetesimal with the Earth was responsible for the formation of the Moon, around 4.5 Gyr bp. Such an impact would have had catastrophic results-melting or vapourising a vast portion of the planet; nothing even vaguely organic could have survived. Indeed, the Moon is bone dry because of the incredible heating that occurred during its cataclysmic formation-and the rotation of Earth was very likely increased to near its present speed by the glancing blow imparted by the impactor. modest bombardment the "warm little pool, with all sorts of ammonia and phosphoric acids, light, heat, electricity, etc. present" envisioned by Charles Darwin as the setting for the original of life would have been boiled into oblivion, or fouled and choked with meteorite debris and ejecta, or stripped clean by planet wide, land scouring tsunamis.

Even so, it is thought that lesser impacts may have been survivable by certain types of ecosystems. Those based on obligate photosynthetic autotrophs-in the top $200 \mathrm{~m}$ of the ocean-would have been highly vulnerable to the effects of large impacts and would have likely been wiped out in the event of such collisions. Chemoautotrophs, perhaps sequestered in the depths of the ocean (where oxidants could perhaps have been produced inorganically) would be less vulnerable to sterilization (although present-day chemoautotrophic ecosystems depend on photosynthesis for their supply of oxidants). Photosynthetic primary producers that could also act as facultative anaerobic heterotrophs would perhaps have been able to find a living at the sea-floor interface, where sufficient oxidant (suphate/sulphite in the ocean) and organic matter (in the ocean sediment) would likely have existed to support such typically small ecosystems ${ }^{2}$.

The course that the evolution of life took depends very much on the actual (rather than probable) extent of the pummeling the Earth received in those early days. Bombardment may have rendered conditions sufficiently inconstant to prevent the early appearance of indigenous life and the planet may have been seeded from outer space (although such a scenario seems unlikely). Alternatively, life may have developed relatively rapidly after the last major (sterilizing) impact on the Earth-certainly there is nothing to rule out such a possibility. Not only that, but life may have evolved several times-indeed our own ancestors may not have been the first representatives of life on Earth. On the other hand, components of the early ecosystem may have been sufficiently robust to withstand such massive impacts ${ }^{2}$. Those organisms existing on the margins of the primordial biosphere, perhaps equivalents of present-day bacteria found deep within the ground, may have been sufficiently well established and resourced to weather such a tens-of-decades long storm-indeed, they may have been selected for by such events.

The new date for evidence of a primordial biosphere do not differentiate between the various models put forward for the possible mode of the evolution of life (all point to the appearance of life prior to $3.85 \mathrm{Gyr} b$ ), although they do suggest that the disruptions to the planet's surface were not sufficient to annihilate the newly formed life there, or disrupt the finely laminated structure of the sedimentary rocks. Assuming the estimates of the late heavy bombardment are accurate, it would seem that life on Earth may have got lucky.

1. Mojzsis, S.J. et al. Nature 384, 55-59 (1996)

2. Maher K.A., \& Stevenson, D.J. Nature 331, 612-614 (1988)

3. Sleep, N.H., Zahnle, K.J., Kasting, J.K. \& Morowitz, H.J. Nature 342, 139-142 (1989) 